\title{
5. Visualization of the Cerebral Vessels by Means of Simplified Percutaneous Cathererization
}

\author{
Komei Nakayama, Hiroyasu Makino, Michio Fukushima, Norio \\ Yamano, Seiichiro Kobayashi and Kozo AKashi \\ Dept. of Surgery, Chiba University
}

Various devices for visualizing the cerebral vessels, which are considered one of the most important and valuable means of diagnosing different diseases, have been reported recently. This method, particularly in visualing the vertebral arteries can be accomplished by the catheterization. However, the ones available for the daily use, brings a rather complicated procedure thus we have made an effort of simplifying the technique which can be used for routine examination. Different size of needles are first devised in order to penetrate the skin with the needle by means of a simple puncture then a polyethylene catheter was introduced into the intraarterial lumen. Ordinary needle has a tendency of dissecting the arterial wall in a transverse direction which can cause bleeding easily. The needles that are to be introduced by this paper will cut the arterial wall in longitudinal direction even they are somewhat difficult in penetrating the skin. This method that we are introducing is simpler to prepare than the ones reported by Seldinger and it does not require a need of air pressure injector which is not available in the Japanese Market.

Various angiograms that have been taken by this method will be introduced during the session.

\section{Localization Studies of a Brain Tumor by Means of Probing Needle G. M. Counter}

\author{
Hideo Sarto, Kosaku Masuda, Syogo Otomo, Makoto \\ KUROSAWA and Yoshiyuki TAKaOKa \\ Dept. of Surgery, Juntendo University School of Medicine
}

Recently, various kinds of radioisotope are used for diagnosis of a brain tumor, and the method and results of positronscanning, areascanning and pointscanning are reported by many investigators.

Present writers tried to decide localization of a brain tumor by Needle G.M.Tube on 46 cases with intracranial lesion at operation. 
Radioactive phosphorus, ${ }^{32} \mathrm{P}$ is administered intravenously about 15 hour before the operation. Counting ratios of brain tumors are compared with norma brain tissues. The localization of brain tumor can be verified in 24 cases of 41 brain tumors-high concentration of ${ }^{32} \mathrm{P}$ can be observed in the case of metastati carcinoma, heterotopic malignant hypophyseal adenoma, astroblastoma, oligoden droglioma, craniopharyngioma and meningioma. Of these, oligodendrogioma anc craniopharyngioma forming cystic tumor have a high concentration of ${ }^{32} \mathrm{P}$ ir cystic membrane.

As to cerebral abscess, is also obtained a high concentration in an abscess membrane. But local diagnosis by this method has not much value in acoustic neurinoma, hypophyseal adenoma and intracranial hematoma. If G.M.-Tube is improved, this test may be useful to localize and to demarcate the brain tumor at operation.

\title{
7. Hormone Replacement Therapy before and after the Operation for Brain Tumor
}

\author{
Miyoshi Urabe, Takashi Tubokawa, Yoshiyuki Matsuda, \\ Yoichi OHTA, and Rokubei UCHINAMI \\ Dept. of Surgery, School of Medicine, University of Kanazawa
}

Using an adrenocortical 11-Beta-Hydroxylase inhibitor (SU-4885), the pituitary reserve was estimated in 13 of the clinical case of the tumor around the third ventricle and in 7 of the other brain tumors.

1) The SU-test revealed distinctly the disturbance of endocrinological circumstance of the brain tumor patients.

2) If the SU-test was done before the operation, the result indicated the extent of the operation of the pituitary tumor regardless of the clinical manifestations, and served for the hormone replacement therapy after the operation.

\section{Late Results of Endocrine Disorders Associated with Parasellar Tumors}

\author{
Katuhiro NiITU, Kokai NishIKAwa and Takashi TAKAHASHI \\ Dept. of Surgery, Iwate Medical College
}

Hypothalamopituitary endocrinologic functions are disturbed and diminished 